\title{
Juvenile Gangrenous Vasculitis of the Scrotum: A Case to Consider
}

\section{Arlene Rodríguez González*, Andrea Orosa Andrada, Jorge Salgado Novoa, Susana Rodríguez Rey, Ana Fandiño Argibay and Venancio Chantada Abal}

Department of Urology, Coruña University Hospital, Spain

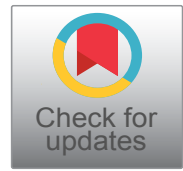

*Corresponding author: Arlene Rodríguez González, Department of Urology, Coruña University Hospital, A Coruña, Spain

\begin{abstract}
Background: Juvenile gangrenous vasculitis of the scrotum is a rare entity that appears in young men, characteristically after an infection of the upper respiratory tract.

Case report: We present the case of a 27 -year-old male patient who presented with fever and scrotal ulcer after pharyngotonsillar symptoms. The differential diagnosis with other entities that present with scrotal ulcer is proposed, including Fournier's gangrene and vascular ulcer secondary to infection by the SARS-CoV-2 virus.

Conclusions: The differential diagnosis of scrotal ulcers is a diagnostic challenge due to its low incidence. Correct identification of the causative entity is essential to determine an adequate therapeutic attitude.
\end{abstract}

\section{Keywords}

Scrotal ulcer, Vasculitis, Gangrene, COVID-19

\section{Introduction}

Juvenile gangrenous vasculitis of the scrotum is an entity of unknown etiology, indolent and with a benign development, which appears in young men as a generally unique, painful and spontaneous necrotic ulcer at the scrotal level, characteristically after an infection of the upper respiratory tract. Fever may be present in this pathology, but no relevant complications have been described in relation to its appearance [1].

Fewer than 25 cases have been reported worldwide since it was first described in 1973 by Pinol, et al [2,3].

\section{Case Report}

We report the case of a 27-year-old man who consulted for a necrotic ulcer at the level of the right hemiscrotum and a fever over 38ㅇ after an episode of pharyngotonsillitis treated with amoxicillin-clavulanic acid. The patient denies risky sexual contacts, local trauma, ingestion of drugs or application of topical substances, as well as associated lower urinary tract syndrome.

The lesion appears as a raised area of a few millimeters two days after the onset of the infectious episode and ulcerates from the fourth day (Figure 1). It presents a rapid evolution until it becomes a necrotic plaque approximately $2 \mathrm{~cm}$ in diameter on the tenth day (Figure 2), when the patient is referred to the emergency room from the out-of-hospital emergency department to exclude Fournier's gangrene.

On physical examination, the patient is pain-free. The ulcer described is observed, not malodorous, without evidence of local infection, without fluctuation or crepitus. The edges are partially detached with no appreciation of bleeding or suppuration. The surrounding scrotal skin is of normal characteristics, as are the penis and perineum. Inguinal lymphadenopathy is not palpable.

On laboratory tests, he presented a slight elevation of C-reactive protein, without leukocytosis or left shift; rest of parameters and urine sediment within normality. The urine culture was negative, while Klebsiella pneumoniae is isolated from the eschar edge culture. Likewise, an urgent scrotal ultrasound is requested and testicular pathology and possible complications such as the presence of gas that could lead to gangrene are ruled

Citation: González AR, Andrada AO, Novoa JS, Rey SR, Argibay AF, et al. (2021) Juvenile Gangrenous Vasculitis of the Scrotum: A Case to Consider. Int Arch Urol Complic 7:077. doi.org/10.23937/2469$5742 / 1510077$

Accepted: July 27, 2021; Published: July 29, 2021

Copyright: (C) 2021 González AR, et al. This is an open-access article distributed under the terms of the Creative Commons Attribution License, which permits unrestricted use, distribution, and reproduction in any medium, provided the original author and source are credited 


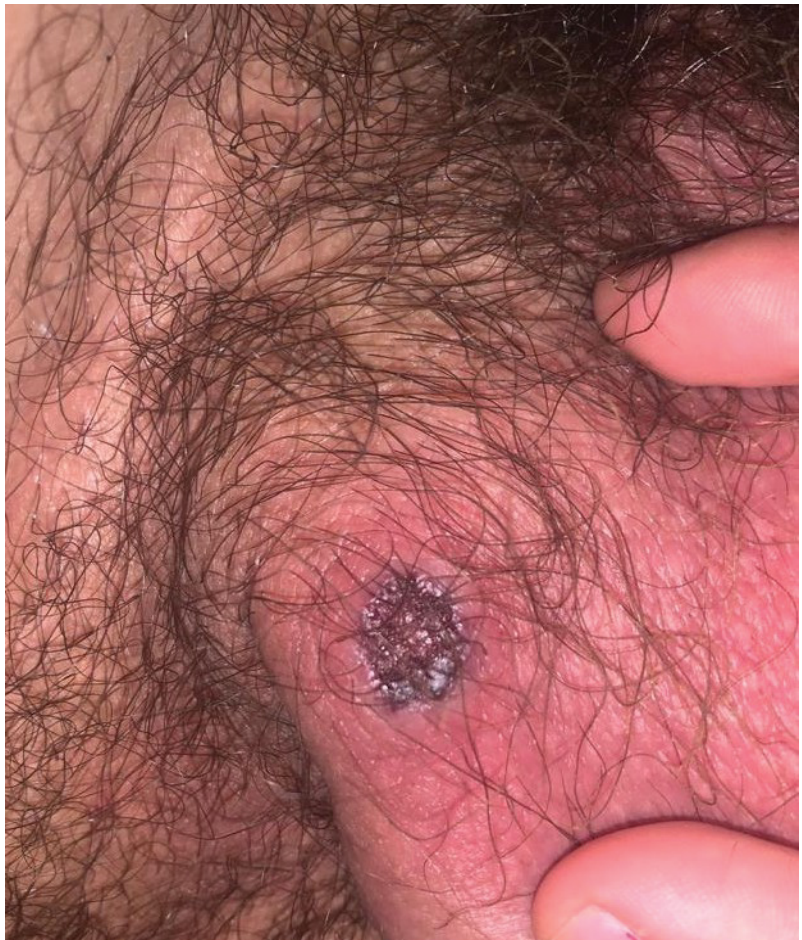

Figure 1: Scrotal injury on the fourth day of evolution.

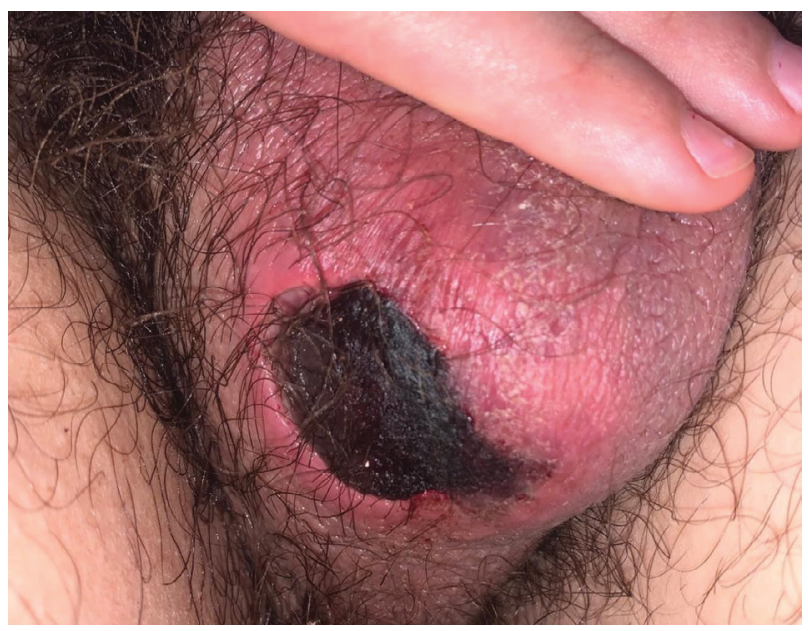

Figure 2: Scrotal injury on the tenth day of evolution.

out (Figure 3). Antibiotic coverage with Ciprofloxacin is started to avoid infection and the patient is admitted to hospital to monitor evolution and continue diagnostic studies.

Being a patient with fever and a history of headache and symptoms of upper respiratory tract infection who attended the emergency room during the global pandemic situation in relation to COVID-19, SARS-CoV-2 infection was ruled out by smear nasopharyngeal for virus PCR.

During admission, the patient is evaluated by the Dermatology Service, which performs a skin biopsy of the lesion, observing areas of ulceration with vasculitis and fibrin thrombi in the pathological anatomy, as well as an acute inflammatory infiltrate with neutrophilic

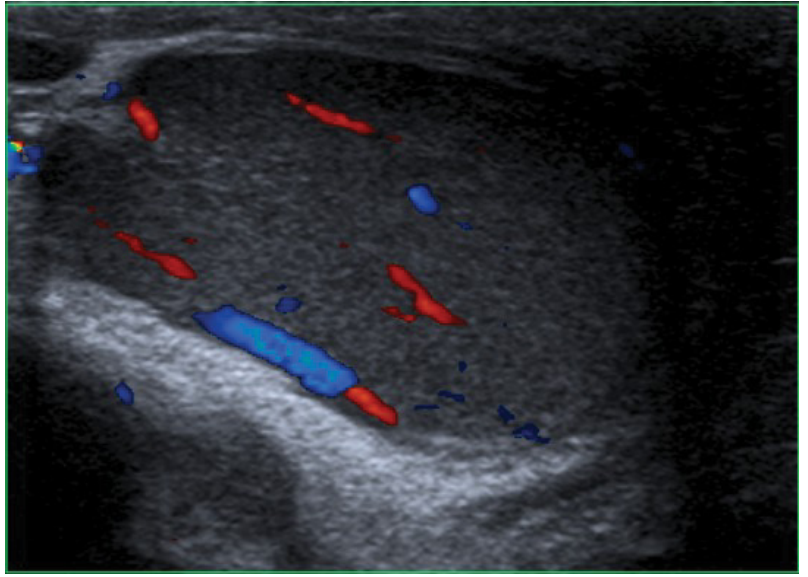

Figure 3: Scrotal ultrasound.

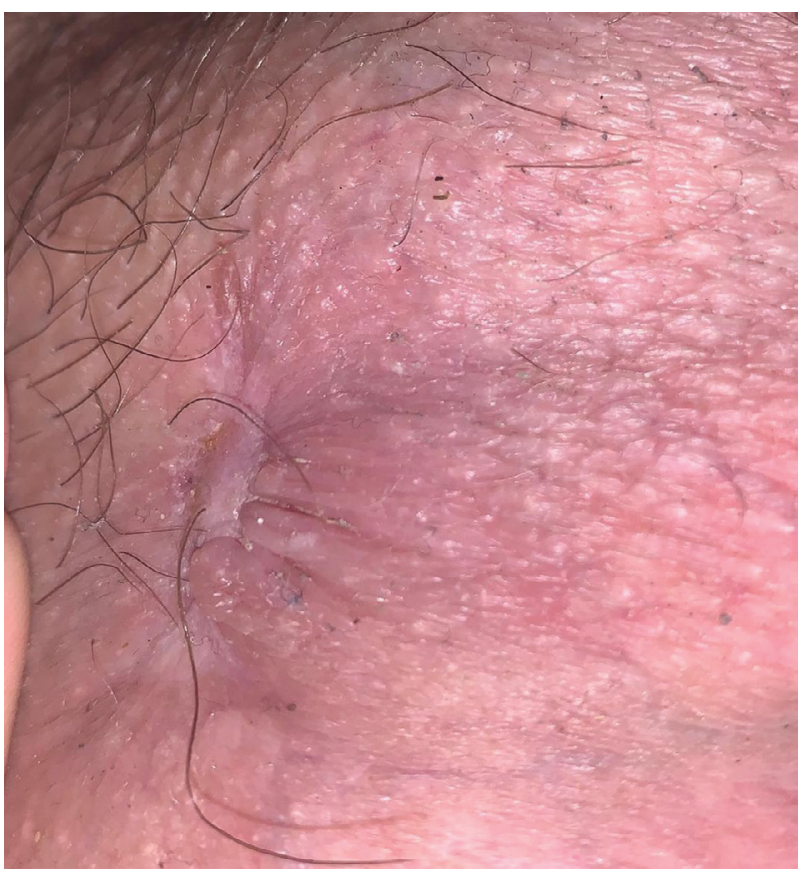

Figure 4: Scrotal injury one month after hospital discharge (45 days after the appearance of the ulcer).

polymorphonuclear leukocytes and fibrin remains. A new culture of the lesion is sent on the third day of admission, being negative at this time. Treponema pallidum, Borrelia burgdorferi and HIV serologies were requested and these were negative.

The patient was discharged due to the good evolution of the ulcer and the febrile syndrome on the fifth day of admission after supportive treatment and oral antibiotics and corticosteroids (complete treatment), without the need for surgical debridement.

In the outpatient follow-up, a good evolution of the ulcer is confirmed (Figure 4), disappearing completely one month after its appearance.

\section{Discussion}

Juvenile gangrenous vasculitis of the scrotum is pathology with few cases described in the literature; 
therefore, its suspicion and a detailed clinical history are key to its diagnosis.

As previously mentioned, it is typical of young male patients, in good health, who present with these ulcers after a pharyngo-tonsillar infection and associated fever. The number of ulcers is usually less than 5 [3], they evolve quickly and are usually small, black and with well-defined raised edges. In histology, the presence of inflammatory infiltrate, as well as necrosis, is common, although not pathognomonic [1].

Therefore, it is essential to carry out an adequate differential diagnosis with other scrotal lesions of similar presentation but that require specific treatment such as those related to sexually transmitted diseases (genital herpes, inguinal granuloma, soft chancre, syphilitic chancre, lymphogranuloma venereum), Lyme disease or much more serious and requiring urgent and aggressive therapeutic measures such as necrotizing fasciitis and Fournier's gangrene. Other pathologies to consider are Behçet's disease, Polyarteritis nodosa and Pyoderma gangrenosum, as well as iatrogenic gangrenes caused by anticoagulants, NSAIDs, antibiotics or skin antiseptics [4].

There is an entity that appears in the female sex called Lipschütz ulcer that has important epidemiological, clinical and microbiological similarities with juvenile gangrenous vasculitis of the scrotum. It is also an entity of benign course, of not well defined etiology and that appears in most cases accompanied by fever and after symptoms of the upper respiratory tract $[5,6]$.

Currently, due to the appearance of COVID-19, we must rule out that a genital ulcer corresponds to an atypical presentation of this entity since cases with the appearance of genital necrotic ulcers with raised edges in COVID-positive patients have already been described in the literature [7].

Regarding treatment, some authors defend that the use of low-dose corticosteroids and antibiotic therapy can reduce the duration of the ulcer and accelerate its resolution, as well as avoid infection problems. In any case, most authors defend that it is a condition that spontaneously disappears approximately one month after its appearance and that it does not require specific interventions or directed therapies except for good local hygiene with daily cures $[1,6]$. Surgical debridement although it is essential when it comes to necrotizing fasciitis, in this entity it is completely unnecessary and can contribute morbidity to the condition.

Despite this, it has been performed in cases in which the diagnosis was not clear or deep involvement was suspected [3].

\section{Conclusions}

Juvenile gangrenous vasculitis of the scrotum is a benign pathology with a favorable evolution that appears in young patients where, despite being a rare entity, proper diagnosis will avoid an excess of complementary tests and possible overtreatment, including debridement surgical.

\section{References}

1. Marcos-Pinto A, Lemos Almeida J, Soares-de-Almeida L, Filipe P (2020) Juvenile gangrenous vasculitis of the scrotum: A rare cause of genital ulcer. Australas J Dermatol 61: e424-e426.

2. Piñol J, Castells A, Lecha M, Ferrando J (1974) A propos de cinq observationes d'une forme pareticuliere de vascularite gangreneuse du scrotum, XIV Congres de L'assoc, Des Derm, Et Syphil, De Langue Francaise, II Vascularites ed: Medicine et Higiene, Geneve, 112-120.

3. Nettleton J, Crawford-Smith $H$, Adimonye A, McMeekin $F$ (2019) Scrotal necrosis and no Fournier's in sight: A rare case of juvenile gangrenous vasculitis. BMJ Case Rep 12: e226530.

4. González González S, Pernas Gómez P, Quintas Martínez R, Fuentes Ceballos EJ, Martinón Sánchez F (2012) Vasculitis gangrenosa juvenil del escroto: Un diagnóstico infrecuente de úlceras genitales. An pediatr 239-240.

5. Jimenez-Cauhe J, Gil-Redondo R, Dominguez-Santas M, Ferrer-Gomez A, Cuevas-Santos J, et al. (2019) Juvenile gangrenous vasculitis of the scrotum: The male counterpart of Lipschütz ulcer? J Eur Acad Dermatol Venereol 33: e459-e461.

6. Chen W, Plewig G (2019) Lipschütz genital ulcer revisited: Is juvenile gangrenous vasculitis of the scrotum the male counterpart? J Eur Acad Dermatol Venereol 33: 1660-1666.

7. Falkenhain-López D, Agud-Dios M, Ortiz-Romero PL, Sánchez-Velázquez A (2020) COVID-19-related acute genital ulcers. J Eur Acad of Dermatol Venereol. 